\title{
Editorial: A Novel Monoclonal Antibody-Targeting Angiogenesis by Inhibiting Secreted Frizzled-Related Protein 2
}

\author{
Julie E. Lang, MD, FACS ${ }^{1,3}$, William W. Tseng, $\mathrm{MD}^{1,3}$, and Irene Kang, MD ${ }^{2,3}$ \\ ${ }^{1}$ Division of Surgical Oncology, Department of Surgery, University of Southern California, Los Angeles, CA; ${ }^{2}$ Division of \\ Medical Oncology, Department of Medicine, University of Southern California, Los Angeles, CA; ${ }^{3}$ University of Southern \\ California Norris Comprehensive Cancer Center, Los Angeles, CA
}

The legendary surgeon-scientist Judah Folkman contributed a wealth of discoveries on the fundamental role of angiogenesis in cancer and other diseases. ${ }^{1}$ This line of investigation led to more than 50 angiogenesis inhibitors being investigated in clinical trials and at least 10 that are currently approved by the US FDA. ${ }^{2}$ Angiogenesis inhibitors work via a variety of mechanisms of action. Some specifically bind to (1) vascular endothelial growth factor (VEGF) or its receptor; (2) other growth factors such as platelet-derived growth factor (PDGF), fibroblast growth factor (FGF), and epidermal growth factor (EGF); or (3) receptors or proteins on endothelial cells, blocking the growth of blood vessels. ${ }^{3,4}$ Angiogenesis inhibitors remain an attractive topic for preclinical development for cancer therapy.

Secreted frizzled-related protein 2 (SFRP2) is a modulator of Wnt signaling that is expressed in the vascular endothelium of the majority of solid tumors. ${ }^{5}$ Courtwright et al. reported that SFRP2 mediates angiogenesis by demonstrating that tacrolimus inhibition of SFRP2 decreased vascular tube formation in vitro and reduced the growth of angiosarcoma xenografts in vivo. ${ }^{5}$ Garcia et al. build upon this work in the article entitled "Development of a novel humanized monoclonal antibody to secreted frizzled-related protein-2 that inhibits metaplastic breast cancer and angiosarcoma growth in vivo". ${ }^{6}$

\section{(C) Society of Surgical Oncology 2019}

First Received: 4 August 2019;

Published Online: 9 September 2019

J. E. Lang, MD, FACS

e-mail: Julie.Lang@med.usc.edu
This study focuses attention to SFRP2 inhibition in angiosarcomas and triple-negative breast cancer, two aggressive malignancies where there exists an unmet need for targeted therapies directed at tumor biology. Targeted therapy efficacy is improved when biomarkers can be found that help identify a responding patient population. It is encouraging that SFRP2 does appear to be strongly expressed in around $70 \%$ of breast cancers as well as many other types of cancers based on a query of The Human Protein Atlas (www.proteinatlas.org). ${ }^{7}$ The authors have found that SFRP2 is broadly expressed across a variety of solid malignancies, both epithelial and mesenchymal in origin. Specifically, high SFRP2 expression is seen in $85 \%$ of triple-negative breast cancers and $100 \%$ of angiosarcomas. ${ }^{5}$ Other investigators have also shown that the family of SFRPs appears to play an important role in osteosarcoma. ${ }^{8}$ Importantly, SFRP2 and its related isoforms are involved in the Wnt pathway, which plays a critical role for many other sarcoma subtypes (e.g. desmoid tumors, synovial sarcoma),${ }^{9-11}$ which suggests potentially even more relevance for this particular group of malignancies.

The authors elegantly demonstrated that their humanized monoclonal antibody against SFRP2 reduced tumor growth and increased apoptosis of SVR angiosarcomas and Hs578T triple-negative breast cancers in vivo. There is definitely a need to demonstrate preclinical efficacy in mice prior to human clinical trials, but the immunodeficient mouse model and the lack of immune response when tested in vitro with healthy human blood argues against the mechanism of action being related to the host immune response. This could be further studied using immunocompetent syngeneic mouse models such as $4 \mathrm{~T} 1$ in BALB/ $\mathrm{c}$ mice to model triple-negative breast cancer. Alternatively, genetically engineered mouse models such as FVB- 
$\mathrm{Tg}(\mathrm{C} 3-1-\mathrm{TAg})$ or the p53 null 'T11' models for triplenegative breast cancer or the Pdgfrb-Cre, Trp53 ${ }^{\mathrm{R} 172 \mathrm{H} / \mathrm{R} 172 \mathrm{H}}$ and $\mathrm{H} 2 \mathrm{~K}$-fos-tg mouse models for angiosarcoma and osteosarcoma, respectively, could be used. ${ }^{12-15}$ These models would need to be tested to see if they express the human target antigen, or otherwise engineered to do so. ${ }^{16}$

Immunotherapy has become a widely used modality in many advanced cancers. The concept of synergy between angiogenesis inhibitors and immunotherapy is of great interest and has been explored by several other groups. ${ }^{17,18}$ Vascular normalization could, in theory, lead to a more effective influx of immune cells necessary to achieve an antitumor response. ${ }^{19}$ Furthermore, the hypothesis that immune checkpoint inhibitors could improve the efficacy of antiangiogenic therapies in cancer is certainly deserving of further investigation. ${ }^{12,20,21}$ Immunotherapies can inhibit the immunosuppressive endothelial barrier via inhibition of programmed death-1 (PD-1) activation. ${ }^{20}$ Endothelial programmed death-ligand 1 (PD-L1) expression has been reported to regulate angiogenesis by directly modulating VEGF receptor 2 (VEGFR2) expression and increasing endothelial proliferation; therefore, PD-1 blockade could have antivascular activity. ${ }^{22}$ This mechanism is in line with emerging evidence that angiogenesis and immunosuppressive responses frequently occur simultaneously physiologically but may be capitalized on by malignancies. ${ }^{23}$

Antagonism of SFRP2 in the vascular endothelium would be expected to inhibit tumor growth, angiogenesis, and tumor migration. Accompanying these expected therapeutic effects would be toxicities. Serious toxicities seen with antiangiogenic therapeutics, such as VEGF inhibitors and multitargeted tyrosine kinase inhibitors, include hypertension, hemorrhage, thrombosis, stroke and/or myocardial infarction, proteinuria, reversible posterior leukoencephalopathy, and endocrine dysfunction. ${ }^{24-26}$ Impaired wound healing via inhibition of migration and proliferation of endothelial cells is also an important described adverse effect. Therapy utilizing monoclonal antibodies have described infusion-related reactions to varying degrees. ${ }^{27}$ Although experience with bevacizumab (a humanized monoclonal antibody to VEGF) has not demonstrated clinically significant hypersensitivity, ${ }^{28}$ the potential for infusion-related reactions with this novel agent should not be ignored. Finally, other described adverse effects include fatigue, gastrointestinal symptoms, hand-foot syndrome, stomatitis, cutaneous toxicity, and hepatotoxicity.

Looking to the history of VEGF inhibition and other angiogenesis inhibitors, clinical benefit has been described with monotherapy of a few agents (e.g. cabozantinib, ramucirumab, sunitinib, sorafenib), and, more significantly, in combination with cytotoxic chemotherapy in a wide range of solid tumors. ${ }^{24,25,29}$ Therefore, determining the efficacy of preclinical and eventually clinical combinations with cytotoxic chemotherapy and even immunotherapy will be useful future strategies. ${ }^{29}$ Important directions moving forward will be to understand mechanisms of resistance to SFRP2 inhibition and examine the role of SRFP2 inhibition in combination with other modalities of therapy (i.e. chemotherapy, radiation, immunotherapy, and other targeted therapies).

Garcia et al. are to be congratulated on optimizing and developing a novel experimental therapeutic agent; however, much work still remains to be done before embarking on clinical trials. Preclinical studies such as pharmacokinetics (PK), toxicokinetics (TK), serum concentrations of treated animals, tissue cross-reactivity, local tolerance, as well as single and repeat dose toxicity must be considered prior to applying to the US FDA for an Investigational New Drug (IND) application. ${ }^{30}$ It is customary that phase I trials include a large spectrum of solid malignancies, including patient populations suitable for treatment with a goal of determining the recommended phase II dose (RP2D) and establishing the safety of the drug in humans. It would be anticipated that the target populations of interest, angiosarcoma and triple-negative breast cancer patients, would be well represented in a phase II trial, in which a preliminary efficacy signal might be detected. The process of drug discovery is an arduous one, but the timeline for clinical trial testing of a novel cancer therapy may take an additional decade. Given that patients with often lethal cancers such as angiosarcomas and triple-negative breast cancers may stand to benefit from this novel therapy, we encourage the authors to go full speed ahead to embark on clinical trials evaluating this monoclonal antibody.

ACKNOWLEDGMENT The project described was supported in part by award number P30CA014089 from the National Cancer Institute. The content is solely the responsibility of the authors and does not necessarily represent the official views of the National Cancer Institute or the National Institutes of Health.

DISCLOSURES Dr. Lang received research support from ANGLE Parsortix, is on the speakers bureau for Genomic Health, and is a scientific advisor to Quantgene. Dr. Kang is on the speakers bureau for Puma Biotechnology. Dr. Tseng has no disclosures to declare.

\section{REFERENCES}

1. Folkman J. Tumor angiogenesis: therapeutic implications. $N$ Engl J Med. 1971;285(21):1182-1186.

2. Chabner BA, Koop CE, Niederhuber JE, Pinedo HM. Homage to Judah Folkman. Oncol. 2008;13(2):205-211.

3. Folkman J. Angiogenesis: an organizing principle for drug discovery? Nat Rev Drug Discov. 2007;6(4):273-286.

4. Cook KM, Figg WD. Angiogenesis inhibitors: current strategies and future prospects. CA Cancer J Clin. 2010;60(4):222-243. 
5. Courtwright A, Siamakpour-Reihani S, Arbiser JL, et al. Secreted frizzle-related protein 2 stimulates angiogenesis via a calcineurin/ NFAT signaling pathway. Cancer Res. 2009;69(11):4621-4628.

6. Garcia D, Nasarre P, Bonilla I, et al. Development of a novel humanized monoclonal antibody to secreted frizzled-related protein-2 that inhibits metaplastic breast cancer and angiosarcoma growth in vivo. Annals of Surgical Oncology. (in press).

7. Uhlen M, Zhang C, Lee S, et al. A pathology atlas of the human cancer transcriptome. Science. 2017;357(6352):eaan2507.

8. Bravo D, Salduz A, Shogren KL, et al. Decreased local and systemic levels of sFRP3 protein in osteosarcoma patients. Gene. 2018;674:1-7.

9. Crago AM, Chmielecki J, Rosenberg M, et al. Near universal detection of alterations in CTNNB1 and Wnt pathway regulators in desmoid-type fibromatosis by whole-exome sequencing and genomic analysis. Genes Chromosom Cancer. 2015;54(10):606615 .

10. Cironi L, Petricevic T, Fernandes Vieira V, et al. The fusion protein SS18-SSX1 employs core Wnt pathway transcription factors to induce a partial Wnt signature in synovial sarcoma. Sci Rep. 2016;6:22113.

11. Vijayakumar S, Liu G, Rus IA, et al. High-frequency canonical Wnt activation in multiple sarcoma subtypes drives proliferation through a TCF/beta-catenin target gene, CDC25A. Cancer Cell. 2011;19(5):601-612.

12. Maroulakou IG, Anver M, Garrett L, Green JE. Prostate and mammary adenocarcinoma in transgenic mice carrying a rat C3(1) simian virus 40 large tumor antigen fusion gene. Proc Natl Acad Sci U S A. 1994;91(23):11236-11240.

13. Herschkowitz JI, Zhao W, Zhang M, et al. Comparative oncogenomics identifies breast tumors enriched in functional tumor-initiating cells. Proc Natl Acad Sci $U$ S A. 2012;109(8):2778-2783.

14. Salter DM, Griffin M, Muir M, et al. Development of mouse models of angiosarcoma driven by p53. Dis Model Mech. 2019;12(7):dmm-038612.

15. Uluckan O, Segaliny A, Botter S, Santiago JM, Mutsaers AJ. Preclinical mouse models of osteosarcoma. Bonekey Rep. 2015;4:670.

16. Podolin PL, Webb EF, Reddy M, Truneh A, Griswold DE. Inhibition of contact sensitivity in human CD4 + transgenic mice by human CD4-specific monoclonal antibodies: CD4 + T-cell depletion is not required. Immunology. 2000;99(2):287-295.

17. Nair S, Boczkowski D, Moeller B, Dewhirst M, Vieweg J, Gilboa E. Synergy between tumor immunotherapy and antiangiogenic therapy. Blood. 2003;102(3):964-971.
18. Lode HN, Moehler T, Xiang R, et al. Synergy between an antiangiogenic integrin alphav antagonist and an antibody-cytokine fusion protein eradicates spontaneous tumor metastases. Proc Natl Acad Sci U S A. 1999;96(4):1591-1596.

19. Jain RK. Normalization of tumor vasculature: an emerging concept in antiangiogenic therapy. Science. 2005;307(5706):58-62.

20. Khan KA, Kerbel RS. Improving immunotherapy outcomes with anti-angiogenic treatments and vice versa. Nat Rev Clin Oncol. 2018;15(5):310-324.

21. Fukumura D, Kloepper J, Amoozgar Z, Duda DG, Jain RK. Enhancing cancer immunotherapy using antiangiogenics: opportunities and challenges. Nat Rev Clin Oncol. 2018;15(5):325-340.

22. Jin Y, Chauhan SK, El Annan J, Sage PT, Sharpe AH, Dana R. A novel function for programmed death ligand-1 regulation of angiogenesis. Am J Pathol. 2011;178(4):1922-1929.

23. Motz GT, Coukos G. The parallel lives of angiogenesis and immunosuppression: cancer and other tales. Nat Rev Immunol. 2011;11(10):702-711.

24. Vasudev NS, Reynolds AR. Anti-angiogenic therapy for cancer: current progress, unresolved questions and future directions. Angiogenesis. 2014;17(3):471-494.

25. Al-Abd AM, Alamoudi AJ, Abdel-Naim AB, Neamatallah TA, Ashour OM. Anti-angiogenic agents for the treatment of solid tumors: Potential pathways, therapy and current strategies-a review. J Adv Res. 2017;8(6):591-605.

26. Chen HX, Cleck JN. Adverse effects of anticancer agents that target the VEGF pathway. Nat Rev Clin Oncol. 2009;6(8):465-477.

27. Lenz H-J. Management and preparedness for infusion and hypersensitivity reactions. Oncol. 2007;12(5):601-609.

28. Reidy DL, Chung KY, Timoney JP, et al. Bevacizumab $5 \mathrm{mg} / \mathrm{kg}$ can be infused safely over 10 minutes. J Clin Oncol. 2007;25(19):2691-2695.

29. Ramjiawan RR, Griffioen AW, Duda DG. Anti-angiogenesis for cancer revisited: Is there a role for combinations with immunotherapy? Angiogenesis. 2017;20(2):185-204.

30. Lynch CM, Hart BW, Grewal IS. Practical considerations for nonclinical safety evaluation of therapeutic monoclonal antibodies. MAbs. 2009;1(1):2-11.

Publisher's Note Springer Nature remains neutral with regard to jurisdictional claims in published maps and institutional affiliations. 